\title{
Du Tertre - vie, œuvre et mission
}

Jacques Du Tertre naît à Calais en 1610. Fils de médecin, il reçoit une bonne éducation et à lâge de 23 ans, poussé par l'aventure, il joint la marine des PaysBas. Avec les Néerlandais, il participe entre autres à une grande expédition au Groenland. Puis, fatigué de la vie en mer, il prend service à terre, toujours dans l'armée néerlandaise, et se distingue notamment au siège de Maastricht en ${ }_{16} 2^{1}$. Deux ans plus tard, Jacques Du Tertre change de carrière et joint l'ordre des Frères Prêcheurs de la congrégation de Saint-Louis. Il entre dans les ordres sous l'égide du père Jean-Baptiste Carré, prend un autre prénom et intègre le Noviciat général à Paris.

En 1640, Jean-Baptiste Du Tertre se trouve de nouveau sur un vaisseau, cette fois envoyé en mission aux Antilles. André Chevillard, un autre Frère Prêcheur qui partira dans les Îles quelques années plus tard, décrit ainsi la compagnie avec laquelle voyage notre missionnaire: «le R. P. de la Marre tres-celebre Docteur de Sorbone, \& un des plus fameux Predicateurs de France [...] Pere Iean de S. Paul Bordelois [...] Pere Michel \& quelques Freres Convers: entre lesquels estoient le bon \& zelé F. Nicolas de Sainctar [...] F. Iacques le Gendre, \& F. Estienne de l'Assomption ${ }^{2} »$. À en croire la notice que les dominicains lui consacrent après sa mort, Du Tertre aurait été un homme «pieux et considéré, prudent, sachant traiter les affaires »; qualités essentielles pour la mission, qui implique des négociations constantes avec les autorités religieuses et séculaires en France, ainsi qu'avec les autorités locales ${ }^{3}$. Or, l'argument principal de Carré au moment de choisir Du Tertre était sans doute son expérience marine. Sa vie militaire rattrape ainsi sa vie religieuse, peut-être contre son propre gré, car l'image qu'il donne de la mer est loin d'être positive. « Ie ne me flate point, ie sçay un peu ce que c'est que de la mer : mais il est constant, qu'humainement parlant, nous ne devions pas demeurer un moment sur l'eau», constate-t-il après avoir décrit tous les maux de mer possibles (1654, tome I : 90). Et pourtant, tout au long de la première période de l'établissement et de la colonisation des Îles, c'est toujours Du Tertre qui est envoyé pour transmettre des

1 Anonyme, Le Père du Tertre, son ouvre et sa vie, АNOM, вІв Sом d/Br/1844, p. 10.

2 André Chevillard, Les Desseins de son Éminence de Richelieu pour l'Amérique, Basse-Terre, Société d'histoire de la Guadeloupe, 1973 [Rennes 1659], p. 44.

3 Ninon Maillard, Droit, réforme et organisation nationale d'un ordre religieux en France: Le cas de l'Ordre des Frères Prêcheurs (1629-1660). Thèse de doctorat, soutenue à Toulouse, 2005, p. 679 . 
messages entre les missions et les autorités en France. Entre 1640 et 1658, il fait cinq allers-retours entre les îles Sous-le-Vent et Paris. Or, son dernier voyage sera différent. Cette fois, il ne se déplace pas en tant que missionnaire; il est envoyé du comte Cérillac pour sonder le terrain de l'île de la Grenade que ce dernier avait l'intention d'acheter (1667, tome I : 504). À son retour définitif des Îles, il est affilié à la province de Saint-Louis à Paris, mais en 1666 - soit l'année avant la parution de la deuxième édition de son Histoire des Antilles - il est envoyé au couvent des Frères Prêcheurs de Toul, en Meurthe-et-Moselle. En 1683, on le rappelle au Noviciat général à Paris, où il meurt quatre ans plus tard.

Les séjours de Du Tertre couvrent toute la période des premiers établissements jusqu'à la consolidation de la colonisation des Antilles françaises dans les années 166o. Le missionnaire a été le témoin oculaire de plusieurs des événements qu'il raconte; s'il ne les a pas lui-même vécus, il tire ses informations de plusieurs sources dites fiables, orales aussi bien qu'écrites. De surcroît, servant principalement en Guadeloupe, il voyage dans toute la région et visite notamment d'autres îles où sont impliqués les Français: Saint-Christophe, Saint-Martin, la Martinique, la Dominique et Sainte-Lucie. La comparaison avec Hérodote que nous propose le critique littéraire Régis Antoine trouve ici tout son sens : aucun autre voyageur ne propose une perspective aussi complète sur les Îles, ce qui en fait la référence principale des historiens de la première colonisation 4 .

\section{Du manuscrit aux éditions}

Le premier texte de la plume de Du Tertre qui ait été conservé est un manuscrit autographe daté de 1648 et intitulé Histoire des isles de la Guadelouppe, Martinique, St Christophle, la Dominique et autres isles circonvoisines en l'Amerique, et des sauvages en icelles ... en l'an $1648^{5}$. Ce manuscrit reste inconnu jusqu'en 1844, quand Augustin Cochin, dans son Abolition de l'esclavage, fait référence à un «manuscrit curieux » acheté à la vente d'une grande collection de livres. Ailleurs dans son ouvrage, Cochin renvoie à l'édition de 1654 de l'Histoire générale des Antilles, mais sans faire de rapport entre celle-ci et le manuscrit trouvé. Au contraire, il l'attribue à un autre dominicain sur lequel nous reviendrons à plusieurs occasions dans cette étude, le père Raymond Breton ${ }^{6}$.

4 Régis Antoine, Les Écrivains français et les Antilles, p. 30.

5 Du Tertre est aussi l'auteur d'un ouvrage religieux, La Vie de Sainte Austreberte, 1659.

6 Augustin Cochin, L'Abolition de l'esclavage: Résultats de l'abolition de l'esclavage, Paris, Lecoffre, 1861, p. 4. 
L'absence de lien entre le manuscrit et le livre de Du Tertre s'explique quand on considère la signature mystérieuse de la préface au manuscrit : «Verdier, missionnaire, a écrit cette histoire de la Guadeloupe en 1648. » (NP) On n’a pas pu identifier ce Verdier, et peut-être s'agit-il, comme le suggère Benoît Roux, d'un nom d'emprunt ${ }^{7}$. Quoi qu'il en soit, les spécialistes s'entendent aujourd'hui pour dire que le manuscrit est de Du Tertre. L'avis au lecteur de l'édition de 1667 confirme d'ailleurs ce constat: Du Tertre y fait référence à un manuscrit incomplet qui circule à Paris vers la fin des années 1640. On le retrouve aujourd'hui en trois exemplaires. Deux d'entre eux se trouvent aux Fonds Marcel-Châtillon de la Bibliothèque Mazarine, dont un est dédié à Raymond Breton. L'autre se trouve à la Bibliothèque Nationale, mais à celui-ci manquent le premier cahier ainsi que les deux derniers chapitres. Le manuscrit complet compte 781 pages et contient 26 dessins de la main de Du Tertre, représentant surtout des plantes, mais on y trouve aussi une illustration d'un four pour préparer le sucre et une autre représentant le «feu des Sauvages». L'auteur divise la relation en cinq parties ; la première est constituée d'un «narré sucsint » $(1648: 7)$ de l'aller et du retour aux Indes ainsi que de ce qui s'est déroulé à la Guadeloupe depuis 1626 et jusqu'à l'an 1643. La deuxième offre une description des tempéraments de la zone torride, de la situation de la Guadeloupe, du « flux et reflux de la mer », et une description de la terre. La troisième est un traité des plantes, suivi par un traité des animaux, qui constitue la quatrième partie. Finalement, la cinquième partie décrit «la façon de faire avec les Sauvages ». (1648:11)

Lauteur garde les grandes lignes de cette organisation lorsqu'il retravaille le manuscrit en livre. Dans le premier tome des deux versions publiées, il se présente d'abord comme chroniqueur de l'histoire française des Îles pour, ensuite, dans le deuxième tome, adopter la posture d'un historien naturel et moral, offrant une description de la région et de ses peuples, tout comme dans le manuscrit. Il semble donc que Du Tertre avait en vue une publication dès la rédaction du manuscrit et que celui-ci n'était pas seulement destiné aux bibliothèques des seigneurs impliqués dans la Compagnie des Isles. Cela dit, les éditions imprimées ont subi des remaniements significatifs depuis la version manuscrite. Il y ajoute des dédicaces et des préfaces, contribuant à placer l'ouvrage dans un contexte de construction de savoir et de réception auquel nous reviendrons plus loin. On peut noter que le style des éditions imprimées est souvent plus vif, et là encore, on note des changements de la première à la

7 Benoît Roux, «Le Pasteur Charles de Rochefort et l'Histoire naturelle et morale des îles Antilles de l'Amérique », Les Indiens des Petites Antilles: Des Premiers peuplements aux débuts de la colonisation européenne, Bernard Grunberg (dir.), Paris, L'Harmattan, 2011, p. 214. L'auteur anonyme de Du Tertre et sa vie est le premier à avoir identifié le manuscrit. 
deuxième édition, indiquant que Du Tertre suit de près l'évolution de l'histoire contemporaine des Antilles ainsi que les publications qui paraissent à ce sujet, notamment dans les années 165o. Surtout, aux versions publiées sont intégrées des illustrations faites par un graveur professionnel et des documents relatifs à l'histoire qu'elles racontent.

Lédition de 1654, intitulée Histoire générale des Isles de Saint-Christophe, de la Guadeloupe, de la Martinique et autres dans l'Amérique, est sortie chez Langlois et dédiée à Achilles II de Harlay8. À part quelques changements mineurs, cette édition maintient la structure du manuscrit, à l'exception près du début - au lieu de commencer avec ses propres voyages, il prend comme point de départ l'histoire de l'établissement depuis 1625 jusqu'à 1645 - et de la dernière partie. Celle-ci comprend maintenant deux chapitres supplémentaires qui manquent au manuscrit, l'un portant sur les habitants français et l'autre sur les esclaves. Le chapitre relatif à la description des Amérindiens est toutefois beaucoup plus long que les deux autres. Les illustrations, faites par Du Tertre lui-même, n'ont pas été reprises dans les livres imprimés. Par contre, l'Histoire générale des Isles de Saint-Christophe, de la Guadeloupe, de la Martinique et autres dans l'Amérique contient des cartes de Saint-Christophe, de la Martinique et de la Guadeloupe. De plus, le lecteur trouve en annexe une concession du Roy qui explique comment les îles sont tombées entre les mains des Chevaliers de Malte, ainsi qu'une traduction « de nos mystères en langue Sauvage », c'est-à-dire le catéchisme, que Du Tertre avait reçue du R. P. Raymond Breton afin de donner au lecteur, et plus particulièrement aux futurs voyageurs, une idée de la langue autochtone.

La version que Du Tertre publie chez Thomas Jolly en 1667, Histoire générale des Antilles habitées par les François, est un livre plus long et plus richement illustré. Le premier volume s'ouvre sur les armes de la famille de Harlay et est toujours dédié à Achilles II de Harlay, alors que le deuxième volume paraît sous la protection de son fils, Achilles III de Harlay. Il comprend 12 estampes en taille-douce, des vignettes et un frontispice, qui se trouvent tous dans le deuxième tome. Cette édition est sans aucun doute la plus complète, non seulement parce qu'elle traite une période plus longue, mais surtout parce qu'elle est la plus élaborée. En même temps que la deuxième édition se présente comme un document historique plus solide s'appuyant sur des références qui sont intégrées au récit, elle est aussi plus littéraire, empruntant des tournures rhétoriques au théâtre, au genre épique et au roman. C'est donc

8 Dans certains livres de cette édition, l'épitre est addressée à Claude Sanguin. Harlay semble pourtant en être le mandataire principal : il est mentionné dans le récit même et on sait qu'il avait le manuscrit du livre dans sa bibliothèque. 
l'édition de 1667 qui constitue l'objet principal de notre analyse. On y retrouve des estampes de Sébastien Leclerc, incluant un portrait des habitants autochtones, deux illustrations des forts français, six planches de naturalia (poissons, insectes, lézards, fruits et plantes) et six illustrations de la vie quotidienne (préparation du manioc, du sucre et du pétun, chasse aux tortues, chasse aux cochons sauvages et visite des Autochtones). Ces illustrations complètent la lecture des longues parties descriptives - et, comme le note Gilles Boucher de La Richarderie, contribuent à renforcer le succès de l'œuvre 9 . L'ouvrage est aussi complété par plusieurs documents - concessions, lettres patentes, lettres de cachet, Commission du Roy, délibérations de la Compagnie, lettres et extraits d'autres relations - rendant encore plus crédible la teneur du contenu. La valeur de l'ouvrage a donc considérablement augmenté et, déjà, la couverture annonce une publication en deux volumes, alors qu'un troisième sortira en 1671, incluant deux tomes supplémentaires. Du Tertre raconte ici la construction et la conservation des colonies françaises durant les années 1650 dans l'objectif explicite de servir d'exemple aux futurs gouverneurs qui, en le lisant, apprendront de l'histoire et éviteront de répéter les erreurs de ceux qui leur ont précédé.

Dans cette édition, l'histoire des Îles occupe un volume entier, soit près de 600 pages. L'augmentation s'explique bien sûr par le fait que cette édition relate une période plus longue - de 1625 jusquà 1664 -, mais aussi par le fait que le missionnaire accumule les documents relatifs aux événements importants, suggérant une orientation vers la politique coloniale au détriment de l'histoire de la mission, qui est plus patente dans l'édition de 1654 . Cela va jusqu'à perturber l'organisation globale de la relation: l'ampleur du premier tome force l'auteur à déplacer le récit de ses propres voyages dans un deuxième volume, ce dont Du Tertre s'excuse dans l'avis au lecteur. Outre ces voyages, on retrouve dans le deuxième volume la même structure de l'histoire naturelle et morale que dans le manuscrit et dans la première édition - traités des airs, des mers, des plantes, des animaux et des hommes, autant Autochtones qu'esclaves et habitants français, exactement comme dans la première édition, mais avec force détails. De plus, les chapitres sur les habitants et les esclaves sont désormais à peu près de la longueur de celui sur les Autochtones, et la traduction du catéchisme en langue locale a été supprimée. La partie topographique a été augmentée et offre un aperçu de toutes les Antilles, y compris des îles qui ne sont pas possédées par les Français. L’auteur tend à étoffer les descriptions, non seulement en vue d'être plus précis, mais également afin de les peindre

9 Gilles Boucher de La Richarderie, Bibliothèque universelle des voyages, Paris, Treuttel et Würtz, 1808, t. VI : 167 . 
plus vivement. Le nombre d'adjectifs augmente, et l'auteur insère des commentaires plus longs, souvent à l'aune des écrits d'autres voyageurs. Il peut s'agir de portraits d'individus auxquels il ajoute plus d'information, d'anecdotes donnant de l'ampleur à un passage ou encore des parties introductives brossant par exemple un tableau général de l'état d'une île.

Plus ou moins ouvertement, Du Tertre plaide dans chacune des éditions pour un renforcement du soutien financier pour les colonies, suggérant que l'impérialisme français correspond à un projet fragile à la première moitié $\mathrm{du} \mathrm{XVII}^{\mathrm{e}}$ siècle $^{10}$. Chacune des versions prend soin de dater la naissance des Antilles françaises à 1625, moment où Pierre Belain d'Esnambuc et Urbain du Roissey débarquent à Saint-Christophe, où se sont déjà établis les Anglais et une colonie de Français. L'année suivante, Richelieu crée la Compagnie de Saint-Christophe dans l'intention d'unir intérêts commerciaux et évangéliques, et le roi accorde le pouvoir à D'Esnambuc et à du Roissey

[...] d'aller peupler, privativement à tous autres, lesdites isles de SaintChristophe et de la Barbade et autres circonvoisines; icelles fortifier, y mener et conduire nombre de prêtres et de religieux pour instruire les Indiens et habitants d'icelles et tous autres en la religion catholique, apostolique et romaine y celebrer le service divin et administrer les sacrements ; $y$ faire cultiver les terres et faire travailler à toute sorte de mines et de métaux ${ }^{11}$.

Le premier établissement ne rapporte pas beaucoup, et la Compagnie de Saint-Christophe fait vite faillite ${ }^{12}$. Elle sera cependant rétablie par Richelieu en 1635 sous le nom de la Compagnie des Isles de l'Amérique. Entre 1620 et 1640, Anglais, Français et Amérindiens parviennent partiellement à se partager en

10 Le but de la propagande était d'inciter au peuplement des Îles. Voir Liliane Chauleau, La Société à la Martinique au XVIIe siècle (1635-1713), Caen, Recherches sur l'histoire des Antilles, 1966, p. 98-99. Selon elle, la deuxième édition de Du Tertre est «plus nuancée dans ses jugements, répondant plus volontiers à ce dessein de publicité », note 1, p. 99 . Une grande partie de la promotion des Îles apparut dans La Gazette de France, ainsi que le montrent Gabriel Debien et Joseph Le Beer, citant un article de 1638 intitulé « Exploits et logement des Français dans l'Isle de Guadeloupe» qui se termine sur l'espoir «que plusieurs François voyans une terre si fertile et qui n'a besoin que d'habitans en scauraient bien faire leur profit ». Propagande et recrutement pour les Iles au XVII e siècle, Notes d'histoire coloniale, vol. xxxiv, 1954, p. 2 o.

11 Cité dans Réal Ouellet (dir.), La Colonisation des Antilles: Textes français du XVIIe siècle, tomes I-II, Paris, Hermann, 2014, p. 11.

12 Voir Eric Roulet, La Compagnie des Îles de l'Amérique 1635-1651, Rennes, Presses universitaires de Rennes, 2017. 
paix le contrôle de certaines îles comme Saint-Christophe, mais ces périodes d'entente ne sont jamais longues. Du Tertre rapporte plusieurs massacres des Amérindiens à Saint-Christophe et plus tard en Guadeloupe et en Martinique. Les guerres se succèdent, les colonies sont ravagées par la famine et les maladies, les Îles passent d'une autorité à l'autre; enfin, le prix du tabac n'est pas stable. La véritable colonisation ne commence qu'à partir de l'année de l'arrivée de $\mathrm{Du}$ Tertre, en 1640. Il ne fait alors plus aucun doute que les Français vont rester sur les Îles et, à partir de ce moment, il s'établit une véritable culture coloniale, partiellement indépendante des autorités en France. Toutefois, la présence française reste précaire jusqu'au 31 mars 1660, jour de signature du traité signé par la France et l'Angleterre avec les Amérindiens, assurant la séparation entre les nations et ne reconnaissant la souveraineté des Autochtones que sur deux îles, la Dominique et Saint-Vincent.

Tout ce processus chaotique d'établissement est reflété dans l'ouvrage de Du Tertre. Sur l'une des cartes insérées de l'édition de 1654, on voit par exemple que la Martinique est divisée entre les Français, qui avaient pris possession de la partie ouest de l'Île, et les Amérindiens, ces derniers occupant la zone à l'est.

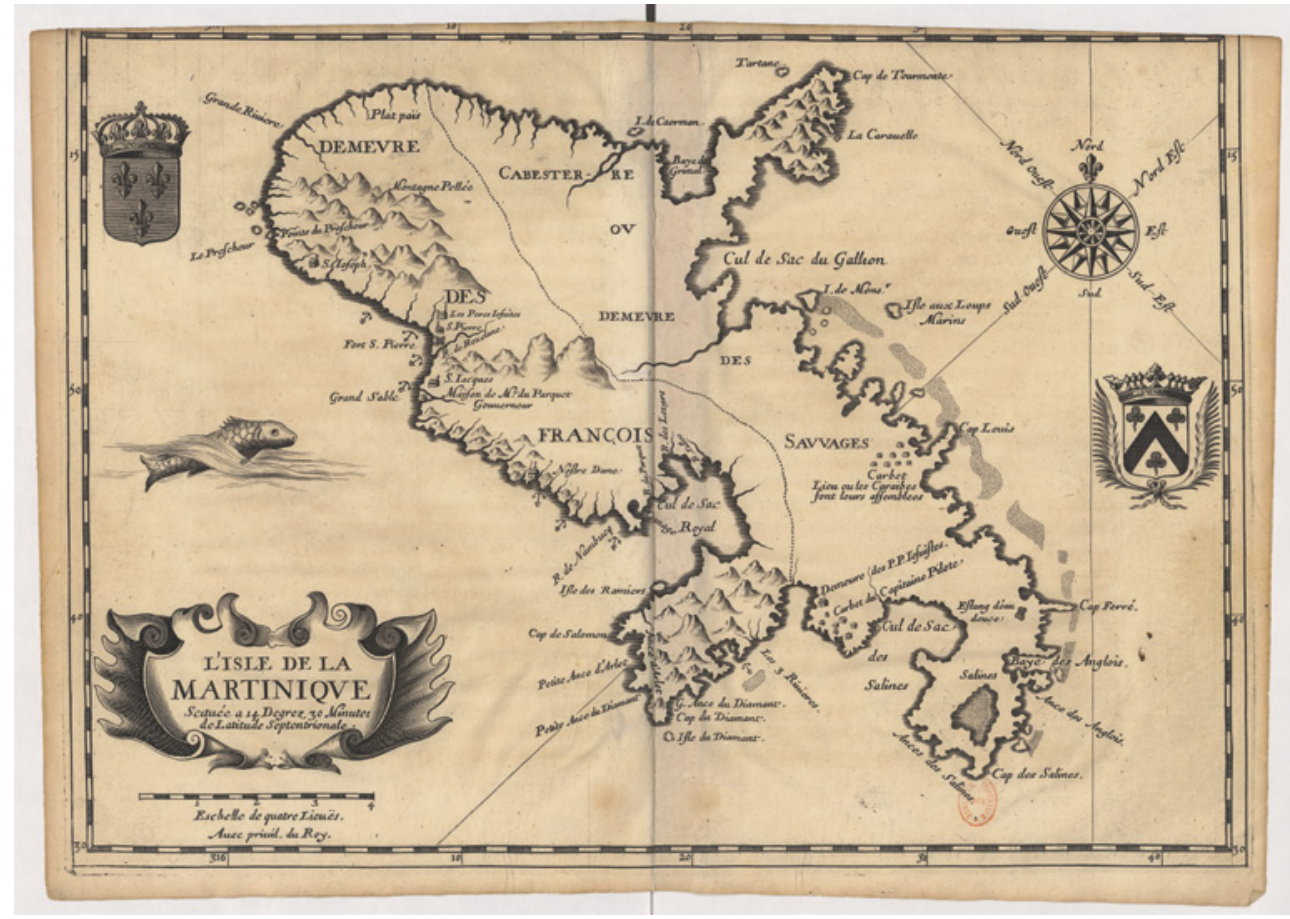

ILLUSTRATION 2 L'Isle de la Martinique

SOURCE GALLICA.BNRF.Fr / BIBLIOTHÈQUE NATIONALE DE FRANCE, DOMAINE PUBLIC 
La raison pour laquelle l'établissement se caractérise par une certaine liberté vis-à-vis des autorités en France est que la guerre de Trente Ans et la Fronde suscitent des préoccupations plus pressantes que celles du contrôle des colonies ${ }^{13}$. Cette liberté partielle due aux événements de l'Europe mène aussi à l'instabilité ; personne ne sait plus qui détient l'autorité. Pendant cette période où les liens entre les Îles et la métropole s'affaiblissent, Philippe Lonvilliers de Poincy de l'ordre de Malte, qui fut nommé lieutenant général de Saint-Christophe en 1638, profite de la situation et commence aussitôt à exercer un pouvoir absolu sur les colonies, jusqu'à sa mort en 166o. Sous sa gouvernance, la Compagnie des Isles de l'Amérique se ruine et on voit se développer entre 1649 et 166o ce que l'historien Philip Boucher appelle « l'ère des propriétaires » ${ }^{14}$ l'époque où la Compagnie vend les îles aux seigneurs propriétaires (de Poincy se charge de Saint-Christophe; Charles Houël prend la Guadeloupe et les îles adjacentes; Du Parquet achète la Martinique, la Grenade et les Grenadines) et où la culture des plantations prend forme. Le règne de de Poincy est sévère et incertain, mais son autorité ne se fait pas seulement au détriment des habitants des Îles. Par exemple, de Poincy fait en sorte que ses sujets soient exempts d'impôts, lesquels faisaient tant souffrir les pauvres en France. En même temps, il les contrôle d'une main de fer. Du Tertre est très ambivalent vis-à-vis de de Poincy dans son ouvrage et rapporte dans l'édition de 1667 des scènes de torture et des exécutions aléatoires qui se seraient déroulées sous son autorité. Du Tertre doute aussi de sa fidélité envers l'Église catholique, en suggérant que ce chevalier de Malte n'hésitait pas à comploter avec les protestants. En outre, de Poincy refuse de reconnaître l'autorité de Patrocles de Thoisy, envoyé par la reine régente et la Compagnie en 1645 . C'est alors que les conflits s'accumulent, risquant d'entraîner les colonies dans une guerre civile.

Voyant pointer la menace d'une guerre civile aux Îles, et sous l'influence de Jean-Baptiste Colbert, Louis XIV change l'orientation de la politique coloniale de la France ; c'est la fin de l'ère des propriétaires et de la longue période d'établissement ${ }^{15}$. Nommé intendant de la Marine par Louis XIV en 1661, Colbert avait déjà créé de grandes compagnies à monopole pour soutenir l'expansion impériale de la France. En 1664, il établit la Compagnie des Indes occidentales et édicte le régime exclusif, lequel impose aux colons de n'avoir de commerce qu'avec la France. À cette époque, le roi envoie Alexandre de

13 Philip Boucher, France and the American Tropics to 1700, p. 67.

14 Ibid., chapitre 4. Voir aussi Frédéric Régent, Les Maîtres de la Guadeloupe: Propriétaires d'esclaves 1635-1848, Paris, Tallandier, 2019.

15 Jacques Dampierre, Essai sur les sources de l'histoire des Antilles françaises, 1492-1664, Paris, A. Picard, 1904, p. xiii. 
Prouville de Tracy pour résister aux Anglais et en finir avec la dominance néerlandaise dans la région. L'année suivante, des séditions de colons se produisent à la Martinique, et l'ordre de Malte vend Saint-Christophe à la Compagnie. Finalement, pour affirmer définitivement son emprise sur les colonies, Colbert fait en sorte que l'administration de celles-ci passe des Affaires étrangères à la Marine, en 1669. L'administration des Îles est maintenant directement liée à la Couronne et se transforme en une «machine coloniale», pour emprunter l'expression de François Regourd et James E. McClellan III ${ }^{16}$.

Les modifications qui interviennent dans la politique coloniale à partir de 166o rendent la sortie inattendue d'un troisième volume, comprenant les tomes trois et quatre, particulièrement signifiante. Dans ce volume, qui n'était donc pas prévu lorsque Du Tertre rédigeait son ouvrage en 1667, Harlay n'apparaît plus comme dédicataire de l'ouvrage, désormais placé sous la protection de Jérôme Bignon, avocat général et maitre de la bibliothèque du roi. L'Histoire générale des Antilles a par ailleurs légèrement changé de cadre générique lorsqu'elle est publiée en 1671 . Au lieu de la présenter comme une relation de voyage ou une histoire naturelle et morale, le missionnaire affirme dans l'avis au lecteur qu'il s'agit d'un traité d'histoire contemporaine, essentiellement appuyé sur des correspondances, des témoignages et des registres. Du Tertre passe donc - en apparence - d'une relation constituée essentiellement sur le témoignage oculaire à un travail exclusivement documentaire. Dans sa pratique, cependant, il se base bien sur son expérience de voyageur et sur les entretiens qu'il a eus avec les témoins des événements qu'il relate dans son livre. Son enquête, ethnographique avant la lettre, s'appuie sur les témoignages qu'il a recueillis au Havre, à Dieppe et à La Rochelle de la bouche des capitaines de navires.

Le volume est divisé en quatre traités. Le premier aborde l'entreprise de $\mathrm{M}$. de la Barre en Cayenne. Le second traité décrit l'établissement de la Compagnie royale des Indes occidentales et l'arrivée de de Tracy aux Îles, et contient une description plus longue de la colonisation de Saint-Domingue et de l'île de la Tortue, ce qui permet à son auteur d'intégrer un portrait des boucaniers et des histoires relatives aux flibustiers. Le troisième s'ouvre sur le récit des actions de de Tracy, raconte la mise en place des offices par la Compagnie et la prise

16 James E. McClellan III et François Regourd, The Colonial Machine: French Science and Overseas Expansion in the Old Regime, Turnhout, Brepols, 2011. Regourd définit ainsi la notion de machine coloniale : «l'ensemble du dispositif gouvernemental et administratif qui, particulièrement depuis Colbert, est impliqué dans les affaires coloniales d'Ancien Régime » dans son article, «Capitale savante, capitale coloniale : sciences et savoirs coloniaux à Paris aux XVII e et XVIII e siècles ", Revue d'histoire moderne et contemporaine,

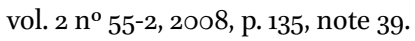


des îles qui étaient contrôlées par les Chevaliers de Malte. Enfin, le quatrième traité est consacré aux soulèvements des habitants contre la Compagnie et à la façon dont de Tracy y met fin; il se termine sur des considérations relatives à l'état de l'Église aux Antilles.

L'enjeu politique du travail de Du Tertre ressort clairement dans ces dernières parties, qui se distinguent à cet égard des deux premiers volumes. Le sous-titre le confirme : «Du changement des Proprietaires des Ant-Isles. De l'Establissement de la Compagnie Royale des Indes Occidentales et son gouvernement jusqu'à la guerre avec les Anglois. » Le livre prend en effet les apparences d'une commission de Colbert. C'est sous son ordre que la Compagnie royale des Indes occidentales fut établie en 1664 contre le gré des colons qui, pour s'opposer à celle-ci, faisaient de la contrebande avec les Néerlandais ${ }^{17}$. Dans sa préface, Du Tertre met la chute de la Compagnie des îles de l'Amérique sur le compte de la mauvaise conduite des hommes à qui les seigneurs impliqués dans la Compagnie se sont fiés, c'est-à-dire, indirectement, les colons et les gouverneurs. (1671:3) C'est leur comportement qui aurait dressé les habitants contre les compagnies. Le but de ces deux tomes supplémentaires, dit-il, est de montrer « le déplorable estat de l'Isle de la Guadeloupe, causé par la division de ses Gouverneurs, \& de ses Proprietaires : celuy de la Martinique, qui estoit gouvernée par des Tuteurs, qui avoient plus de soin de leurs propres interests, que de celuy de leurs pupils \& du bonheur de cette Isle ... » (1671:3) Il s'attaque à la tutelle de de Poincy et des Chevaliers de Malte et ne semble défendre que le commandeur de Sales de Saint-Christophe, avant de constater «qu'elles [les Îles] auroient toutes infailliblement peri dans cette derniere guerre, si elles n'avoient changé de main ». (1671:4) La guerre à laquelle il fait référence est celle contre les Anglais qui mène à la perte de Saint-Christophe, d'Antigua et de Montserrat aux Anglais dans la paix de Breda en 1667. Il est donc profondément fidèle au roi et à la politique coloniale de Colbert, grâce à qui « les affaires y ont tellement changé de face, par l'union qu'il a fait de leur direction à celle de cinq grosses fermes en France, que le commerce n'y peut jamais estre plus asseuré, ny en meilleure posture». (1671:5)

Tout au long de sa carrière, Du Tertre ne s'est jamais départi de sa loyauté vis-à-vis de la monarchie, en ce qui concerne les questions coloniales. Il se montre cependant ici ouvertement critique contre les autorités des Îles (gouverneurs, colons et seigneurs des compagnies). Le changement d'attitude est significatif en ce qu'il reflète la centralisation de la politique coloniale. Il se peut aussi que Colbert soit lui-même intervenu au niveau de la rédaction, ce

17 Philip Boucher, France and the American Tropics to 1700, p. 83 . 
que suggère l'auteur anonyme de La Vie du Père Du Tertre ${ }^{18}$; il faut rappeler que Du Tertre écrit ces derniers volumes à partir de Toul, où on l'avait envoyé pour avoir contesté son supérieur. On peut imaginer que l'éloge de la politique de Colbert est une manière de se lier aux puissants qui depuis Paris pourraient lui éviter l'exil ; une stratégie qui se révélera peu payante : il ne sera renvoyé à la capitale qu'en 1683 .

\section{Les dominicains aux Îles}

Quand Richelieu fonde en 1635 la Compagnie des Isles de l'Amérique, il y établit du même coup l'ordre des dominicains par l'envoi en mission de Pierre Pélican (supérieur), de Raymond Breton, de Nicholas Bruchet de Saint-Dominique et de Pierre Griffon de la Croix, lesquels débarqueront en Guadeloupe. Les Frères Prêcheurs sont donc présents dès le commencement de la colonisation française, ce qui place Du Tertre dans une position privilégiée pour raconter l'aspect chaotique et difficile de l'établissement aux Îles.

Lidée de mission est présente dans la doctrine chrétienne, on le sait, depuis que le Christ enjoint à ses disciples de répandre la Bonne Nouvelle à toutes les nations, mais elle acquiert son sens moderne en français à l'époque de la campagne française pour la colonisation des Amériques. Désormais, le mot «mission » implique des significations aussi bien juridiques que théologiques ${ }^{19}$. Par sa présence même, le missionnaire est l'émanation d'une hiérarchie. Il descend de l'autorité suprême et va à la rencontre des infidèles - ou plutôt descend au niveau des infidèles - pour assurer l'application d'une loi. Dans son étude magistrale Croire et faire croire: Les Missions françaises au XVII e siècle, Dominique Deslandres rapproche la mission coloniale de celle qui s'effectue en France à la même époque : ces missions, externes aussi bien qu'internes, reflètent selon Deslandres le désir de la Couronne de redonner son unité au royaume après les déchirements des guerres de religion ${ }^{20}$. Alors que les missions travaillent

18 Anonyme, La vie du Père Du Tertre, « Comme il avait à y raconter des faits en quelque sorte contemporains, il n'est pas surprenant que de hautes personnalités qu'il ne nomme pas, probablement Colbert lui-même, lui aient fait supprimer à l'impression des passages qui paraissaient trop sincères pour ne pas déplaire aux puissants du jour », p. 16 .

19 Marcel-Antoine Henry, Entrée « Mission », Encyclopaedia universalis France, Paris, 1985, p. 353 .

20 Dominique Deslandres, Croire et faire croire : Les Missions françaises au XVII e siècle, Paris, Fayard 2003. En France aussi bien que dans les colonies, écrit Deslandres ailleurs, « une même logique était à l'œuvre : celle de l'intégration sociale de populations très diverses fondées sur l'adhésion à un message religieux », «Indes intérieures et Indes lointaines: 
à l'unité de la nation, l'Église étend quant à elle sa gloire en implantant la foi chrétienne dans des contrées lointaines. Elle s'engage ainsi dans une politique double, visant à la fois à l'unification et à l'expansion, dans le but de reconstruire la France et de fortifier la foi catholique.

La représentation de l'étranger, chez Du Tertre comme chez d'autres missionnaires, est intimement déterminée par cette fonction. Aux yeux des missionnaires, les paysans français sont considérés comme des sauvages, aussi incultes sinon davantage que les Amérindiens. Empruntons une citation du contexte canadien pour illustrer ce propos: «Je compare volontiers nos sauvages avec quelques villageois, pource que les uns et les autres sont ordinairement sans instruction », écrit le jésuite Paul Le Jeune dans sa Relation de la Nouvelle France de $1634^{21}$. Les Amérindiens ont sur les paysans français cet avantage de pratiquer ce que les missionnaires voient comme une sorte de religion naturelle, et qui suscite parfois leur intérêt. Du Tertre suit sur ce point l'une de ses références majeures, le dominicain espagnol Bartolomé de Las Casas $^{22}$, présentant les Amérindiens comme naturellement enclins à embrasser la foi catholique, leur façon de vivre les prédisposant davantage à suivre un régime strict et à supporter la souffrance, même sans la promesse d'un paradis après la mort. (1667, tome II : 413) Il en va de même pour les esclaves africains qui, à en croire Du Tertre, sont plus faciles à convertir que les Autochtones. Ils sont directement touchés par Dieu et, une fois convertis, précise Du Tertre, «pratiquent les vertus, \& en exercent les œuvres », et affirme aussi, « avec verité, qu'ils y vivent plus Chrestiennement dans leur condition, que beaucoup de François ». (1667, tome II : 502) L'étranger se présente donc chez Du Tertre comme étant apte à se rapprocher des pratiques culturelles françaises, et ce n'est pas nécessairement dans son rapport à l'autre que la mission externe se distingue de l'interne. La différence est plutôt d'ordres spatial et politique: aux Amériques les missionnaires participent, qu'ils le veuillent ou non, à la conquête de territoires et à l'esclavage. Intégrer la religion dans l'espace étranger permet de faire littéralement traverser l'Atlantique à la France et de la transplanter aux Antilles - geste métonymique qui affirme de manière frappante le lien ambigu entre mission et colonisation ${ }^{23}$.

le modèle français d'intégration socio-religieuse au XVII e siècle », La France-Amérique (XVI ${ }^{e}$-XVIII ${ }^{e}$ siècles), Frank Lestringant (dir.), Paris, Honoré Champion, 1998, p. 369.

21 Cité par Dominique Deslandres, Croire et faire croire, p. 306.

22 La Relacion de Las Casas fut traduite en français sous le titre Histoire admirable des horribles insolences et cruautez et tyrranies exercées par les Espagnols ès Indes Occidentales, par Jacques de Miggrode, Paris, Gabriel Cartier, 1582.

Georges Goyau, Missions et missionnaires, Paris, Librairie Bloud \& Gay, 1931, p. 68-69. 
Les missionnaires dominicains établis aux Antilles sont tous plus ou moins liés au Noviciat général et choisis pour la mission par le père Jean-Baptiste Carré $^{24}$. Le Noviciat général est à cette époque un lieu où l'observance religieuse prime sur l'érudition et le savoir. Mais la première génération des missionnaires apporte aussi un autre héritage : le père Raymond Breton, qui sera la référence la plus importante pour Du Tertre, et Nicolas de la Marre, parti en mission en même temps que ce dernier, ont tous les deux fait leurs études à la Sorbonne, où ils ont également enseigné ${ }^{25}$. La notice consacrée au père Breton par le R. P. Feüillet, lui aussi missionnaire et qui le connut pendant son séjour aux Îles, laisse entendre que le développement du savoir était au cœur de l'expérience missionnaire aux Antilles. C'est grâce à l'arrivée de Du Tertre et des autres missionnaires en 1640 que Breton peut finalement abandonner les fonctions de prêtre qu'il remplit auprès des habitants français en Guadeloupe pour se consacrer à son objectif principal, l'évangélisation des Amérindiens ${ }^{26}$. Breton reste 12 ans auprès des Autochtones à la Dominique, et c'est surtout son travail sur le vernaculaire «Caraïbe » qui deviendra essentiel à tous ceux qui écriront sur les Îles ${ }^{27}$. Selon lui, ces hommes s'identifient comme des Callínagos, mais les missionnaires utilisent surtout la dénomination générique «Sauvages», sans doute pour ne pas brouiller un public qui s'intéressait peu

24 Ninon Maillard, Droit, réforme et organisation nationale d'un ordre religieux en France, p. 693. Pour une liste complète de tous les dominicains à la Guadeloupe et à la Martinique entre 1535 et 166o, voir Bernard Grunberg, Benoît Roux et Josiane Grunberg (dir.), Missionnaires dominicains vol. 1, Paris, L'Harmattan, 2016.

25 Sylvain Auroux et Fransisco Queixalos, « La première description linguistique des Antilles françaises: le Père Raymond Breton (1609-1679) », Naissance de l'ethnologie?, p. 109. Sur la formation du savoir des Frères Prêcheurs, voir Roger French et Andrew Cunningham, Before Science: The Invention of the Friars' Natural Philosophy, London \& New York, Routledge, 1996, notamment le chapitre 8.

26 Voir Benoît Roux, «Le prêtre et le Callínago: les missions françaises auprès des Amérindiens des Petites Antilles au XVII ${ }^{\mathrm{e}}$ siècle», Le Contrôle de la vie religieuse en Amérique, Bernard Grunberg (dir.), Paris, L'Harmattan, 2008, p. 98. Voir aussi Louis Fournier, Le V.P. Raymond Breton de l'ordre des Frères Prêcheurs, profès du couvent de Beaune, missionnaire aux Antilles : 1609-1679, Dijon, Bulletin d'histoire et d'archéologie religieuse du diocèse de Dijon, juillet-août, 1895, p. 19.

27 Raymond Breton, Relations de l'île de la Guadeloupe, Basse-Terre, Éditions de la société d'histoire de la Guadeloupe, 1978. Pour un compte-rendu de son séjour aux Antilles et de son travail auprès des Amérindiens, voir l'introduction à l'édition de son dictionnaire présentée et annotée par le celia et le Gerec, Sybille de Pury, «Le Père Breton par lui-même », Dictionnaire carä̈be-français, Paris, Karthala, 1999 [1665]. Raymond Breton, Grammaire Caraïbe, Auxerre, Gilles Bouquet, 1667 ; Petit catechisme ou Sommaire des trois premieres parties de la doctrine chrestienne, Auxerre, Gilles Bouquet, 1664. 
aux spécificités de ces hommes ${ }^{28}$. Du Tertre paraît pourtant sensible à la différence entre les peuples, parlant à l'occasion de «nos Caraïbes », ou des Galibis, des Callínagos, des Tainos, des Arawaks, mais il utilise aussi le terme péjoratif «cannibales».

La conversion des Autochtones représente seulement un des objectifs de la mission antillaise. Il est tout aussi important de veiller sur l'ordre et le respect des traditions chrétiennes à l'intérieur de la communauté française. Les fonctions des missionnaires vont ainsi alterner suivant l'évolution des colonies. $\mathrm{Au}$ début de l'établissement, l'Église coopère étroitement avec la Couronne et les compagnies. Cela explique en partie la prédominance de l'ordre des Frères Prêcheurs aux Antilles, contrairement à d'autres régions colonisées et non colonisées, notamment la Nouvelle-France, où s'engagent surtout des jésuites. En fait, les premiers prêtres aux Antilles sont des séculiers, suivis aussitôt par les capucins, mais ce sont les dominicains qui s'imposent dans les Îles ${ }^{29}$. Selon la version officielle - et on reviendra là-dessus dans la troisième partie -, le choix des Frères Prêcheurs repose sur le martyre subi par six dominicains espagnols aux mains des Callínagos ${ }^{30}$. En réalité, le succès relatif de la mission dominicaine aux Îles est le résultat d'une entreprise politique plus que d'un choix spirituel. Selon Boucher, Richelieu, moins motivé par son ardeur évangélique à vouloir sauver les âmes des Amérindiens que par la politique, envoie des missionnaires aux Îles avec les compagnies dans l'intention de se lier au pape qui, lui aussi, craint une Espagne trop forte ${ }^{31}$. Avec le Vatican de son côté, Richelieu peut ensuite faire taire les dévots catholiques français qui critiquent son soutien aux pays protestants contre l'Espagne dans la guerre de Trente Ans. La raison pour laquelle il choisit les dominicains est qu'il fut personnellement investi dans le développement de l'ordre au Faubourg Saint-Jacques, mais il

28 Il est, comme le souligne Peter Hulme et Neil Whitehead, impossible de savoir aujourd'hui comment les Autochtones s'identifiaient eux-mêmes. Hulme et Whitehead choisissent, dans l'introduction de l'anthologie Wild Majesty: Encounters with Caribs from Columbus to the Present Day, Oxford, Calderon Press, 1992, la dénomination « Carib» non pas parce qu'elle serait correcte, mais parce qu'elle est ouverte à l'identité de ces différents groupes. Ces dernières décennies, l'archéologie caribéenne a pu mieux tracer les cultures autochtones, leurs établissements, mouvements et échanges; voir Corinne L. Hofman et Anne van Duijvenbode (dir.), Communities in Contact: Essays in Archaeology, Ethnohistory and Ethnography of the Amerindian Circum-Caribbean, Leiden, Sidestone Press, 2011.

29 Georges Goyau, Missions et missionnaires, p. 8o.

$30 \quad$ Ibid., p. 82.

31 Philip Boucher, France and the American Tropics to 1700, p. 68. Du Tertre évoque la dégradation morale des prêtres séculiers ; voir l'Histoire générale des Antilles, tome I, 1667 : 71-72. 
semble que le Père Carré aurait préféré garder ses missionnaires à Paris ${ }^{32}$. Quoi qu'il en soit, Richelieu demande donc au pape l'autorisation de mettre tous les missionnaires aux Antilles directement sous la tutelle ou la « conduitte, juridiction et autorité » du supérieur du Noviciat général de Paris et que «tous les biens, possessions, maisons qu'ils auront seront censés, unis et incorporés audict Noviciat $»^{33}$. Désirant des « personnes dont la croyance soit aussy irréprochable que les mœurs, et la piété aussy signalée que le courage », Richelieu préférait les Frères Prêcheurs pour «la réforme de leur ordre » et « à cause de la bonne odeur de leur sainteté et austérité de vie $»^{34}$.

La structure centralisée de l'ordre dominicain, qui concentre toute l'autorité dans les mains du prieur, le père Carré, bien que peu favorable au travail concret de la mission, permet en théorie à ses supérieurs de mieux surveiller cette dernière. Ce contrôle s'avère cependant difficile à maintenir en raison de la distance entre Paris et les Îles. De même que l'administration coloniale souffre du décalage temporel entre l'exécution d'un décret et sa réalisation dans les Îles, le Noviciat ne parvient pas à gérer toutes les affaires de la mission en l'absence d'un contact direct avec les missionnaires - un voyage transatlantique prenant plusieurs mois. De ce fait, la surveillance effective de la mission revient plutôt aux autorités politiques laïques ${ }^{35}$.

Cela dit, il n'est pas insignifiant que l'ordre centralisé et hiérarchique de la mission dominicaine vienne appuyer l'autorité du roi sur les Antilles. Parfois, le Noviciat s'allie avec les autorités séculaires contre ses missionnaires. Lorsque Breton critique sévèrement la guerre menée par les Français contre les Autochtones, le père Carré le presse de revenir en France, prétextant qu'il aurait besoin de repos ${ }^{36}$. En réalité, ce sont les gouverneurs qui veulent éloigner Breton des Îles, puisque la mission empêche l'expansion territoriale ; Du Tertre insère par exemple une lettre dans laquelle de Poincy s'oppose au projet de partir en mission à la Dominique. (1667, tome I : 200) Malgré ces tensions entre le Noviciat, les gouverneurs et les missionnaires, Breton, Du Tertre et les autres sont indispensables à la gestion des colonies et jouent un rôle essentiel à l'établissement dans la mesure où leur présence parmi les Autochtones facilite les rapports entre les Français et les Amérindiens. La structure fondamentale de

\footnotetext{
32 Eric Roulet, La Compagnie des Îles de l'Amérique, p. 10o. Pour le scepticisme de Carré, voir p. 102 et 419.

33 Cité par Dominique Deslandres, Croire et faire croire, p. 691.

34 Ibid., p. 69o. Benoît Roux affirme cependant que Louis XIII préférerait les capucins aux dominicains. Cf., « Le prêtre et le Callínago », p. 83 .

35 Ninon Maillard, Droit, réforme et organisation nationale d'un ordre religieux en France, p. 691.

36 Louis Fournier, Le V.P. Raymond Breton, p. 15-16.
} 
la mission n'est donc jamais remise en cause, et la critique des missionnaires contre le Noviciat ne concerne pas l'organisation même de la mission ni son affiliation politique ${ }^{37}$. Par l'entremise des Frères Prêcheurs, le pouvoir royal cherche un moyen d'étendre son emprise sur les compagnies des Indes, avec lesquelles la mission communique directement. Et cela surtout pendant les années 1640 et 166o, lorsque les rapports avec la métropole sont fragiles. Aussi, le prieur du Noviciat négocie-t-il toujours directement avec les compagnies si la mission a besoin de soutien ${ }^{38}$.

La stratégie employée par la mission pour garder son mandat peut en effet expliquer le caractère politique des relations d'un missionnaire comme $\mathrm{Du}$ Tertre. Doris Garraway note à juste titre :

Il est remarquable que Du Tertre arrive à réconcilier un récit d'abus colonial français et d'échec missionnaire avec une prise de position procoloniale et promissionaire en présentant l'histoire comme un conte moral dans lequel les colons français sont mis à l'épreuve, jugés et punis par la divinité qu'ils servent ${ }^{39}$.

Les réflexions de Garraway ne portent en fait que sur l'édition de 1654, qui raconte l'établissement et la première colonisation avant l'expulsion des Amérindiens. Dans ce contexte, l'auteur met effectivement l'accent sur le travail évangélique de l'ordre des Prêcheurs et sur la condamnation morale de la mauvaise gouvernance. Les gouverneurs, qui dirigent mal leurs colonies et maltraitent les Amérindiens et les engagés, sont ainsi montrés comme punis pour avoir manqué au respect qu'ils doivent aux témoignages de la création divine. Cette dimension morale, certes toujours inspirée des thèses de Las Casas, s'explique pourtant aussi par le contexte de la publication. L'année qui précède la sortie du livre, Breton se rend à Paris pour quémander la protection du roi contre les gouverneurs qui s'emparaient des terres des missionnaires ${ }^{40}$. La rhétorique moraliste de Du Tertre s'inscrit dans la même stratégie : la survie de la

37 Ninon Maillard, Droit, réforme et organisation nationale d'un ordre religieux en France, p. 705 .

$38 \quad$ Ibid., p. 691.

39 Doris Garraway, The Libertine Colony: Creolization in the Early French Caribbean, Durham NC, Duke University Press, 2005, «What is fascinating is that Du Tertre managed to reconcile a story of French colonial abuses and missionary failure with a procolonial, promissionary stance by telling history like a morality tale in which the French colonists are challenged, judged and disciplined by the deity whom they serve», p. $5^{2}$. Notre traduction.

Louis Fournier, Le V.P. Raymond Breton, p. 19. 
mission en dépend. Ces efforts aboutissent, puisqu'en 1662, le roi remet aux missionnaires la terre que les gouverneurs leur avaient ôtée. Mais des circonstances externes expliquent aussi pourquoi les droits de la mission sont mis en avant dans l'édition de 1654. Sans doute à cause des tensions entre pouvoir spirituel et pouvoir temporel, entre la dépendance de l'Église vis-à-vis des puissances politiques et les velléités d'intervention des religieux dans la politique locale des régions auxquelles ils sont affectés, les missionnaires sont, à partir de 1659 , interdits de toute activité politique ${ }^{41}$. Aussi voit-on la dimension moralisante s'atténuer dans la deuxième édition, où l'histoire de la mission est moins centrale. De plus, on a vu que Du Tertre s'exprime dans le dernier volume en tant que porte-parole du pouvoir royal contre les compagnies et les gouverneurs qui rivalisent pour la domination des îles.

En effet, l'évangélisation en soi s'avère un échec. Breton avoue n'avoir converti que quatre personnes lors de son séjour à la Dominique ${ }^{42}$. L'écart entre les habitants français et les Amérindiens s'accroît à partir de 166o, lorsque ces derniers sont expulsés de la Martinique, de la Guadeloupe et de Saint-Christophe, et habitent désormais à la Dominique et à Saint-Vincent. Dès lors, les Autochtones ne représentent plus une véritable menace pour les colonies, et les missionnaires n'ont plus à négocier entre Français et Amérindiens. En revanche, il leur est dévolu un rôle plus conservateur : ils veillent sur l'ordre des colonies, instruisent les habitants et s'occupent de la conversion des esclaves. Effectivement, comme on le verra, dans les rares passages où Du Tertre évoque son rôle de missionnaire, il le fait essentiellement à propos de l'aide qu'il apporte aux habitants de la colonie. Lui et les autres missionnaires érigent des croix, établissent des paroisses, disent la messe, portent soin aux malades et protègent les habitants contre des supérieurs abusifs - toutes opérations qui soutiennent létablissement de la colonie. Cela explique en partie le choix de proposer une histoire des mœurs des habitants et de la nature des Antilles; une histoire de leur mission spirituelle fidèle à la réalité sera bien pauvre ${ }^{43}$. $\mathrm{Si}$ le récit de mission dominicain dépasse en ampleur les relations jésuites dans le contexte antillais, c'est entre autres parce qu'un auteur comme Du Tertre fait le choix de ne pas uniquement parler de mission, mais d'explorer la géographie et de faire l'inventaire de la naturalia, tout en s'engageant ouvertement dans le projet colonial.

41 Philip Boucher, France and the American Tropics to 1700, p. 141.

42 Sylvain Auroux et Fransisco Queixalos, « La première description linguistique », p. 112.

43 C'est aussi la thèse de Benoît Roux dans « Le prêtre et le Callínago », p. 81-82 et p. 101. 
Tous les textes imprimés de la première période coloniale française aux Antilles sont écrits par des missionnaires, à une exception près : Histoire et voyages des Indes occidentales, rédigé par l'engagé Guillaume Coppier et publié à Lyon chez Jean Huguetan en $1645^{44}$. Les débuts difficiles de l'établissement français sont alors à la fois bien et mal documentés ${ }^{45}$. Contrairement aux Anglais et aux Néerlandais qui n'avaient pas d'ambition évangélique, les colons français sont accompagnés par des missionnaires dès 1633, d'Esnambuc amenant avec lui le capucin Hyacinthe de Caen qui, deux ans plus tard, sera accompagné de missionnaires dominicains qui décrivent les pays et leurs habitants et documentent aussi la campagne française aux Amériques ${ }^{46}$. Or, comme la France entre dans la guerre de Trente Ans la même année, Richelieu, pour éviter d'aggraver le conflit avec les Espagnols, interdit la publication de récits portant sur l'établissement. Ce n'est qu'après la disparition de Richelieu et avec la signature du traité de Westphalie en 1648 que la plupart des relations des débuts des Antilles françaises sont publiées ${ }^{47}$. Vu dans l'ensemble de la production viatique du siècle, le voyage aux Amériques représente un phénomène marginal, l'Orient attirant davantage l'attention du public français ${ }^{48}$.

44 Guillaume Coppier, Histoire et voyages des Indes occidentales, Lyon, Jean Huguetan, 1645. Il existe un corpus intéressant de textes inédits qui ont été découverts, mais qui sont moins pertinents pour comprendre le contexte textuel de la relation de Du Tertre. Voir les deux volumes édités par Réal Ouellet, La Colonisation des Antilles. Parmi les relations importantes non publiées, mentionnons celle du père Pacifique de Provins, qui passe plus de six ans aux Îles, Relation des Iles de Saint-Christophe, Gardelouppe et la Martinique; un texte rare portant sur le sort des femmes, écrit par Madame Léonor de La Fayolle, Relation de ce qui s'est passé à l'arrivée des filles de Saint-Joseph en l'Amérique 1643 et 1644 ; et un manuscrit écrit par un flibustier inconnu, «l'Anonyme de Carpentras », édité par Jean-Pierre Moreau sous le titre Un flibustier français dans la mer des Antilles 1618-1620, Paris, Seghers, 1990 .

45 Pour une excellente chronologie de la colonisation des Antilles, voir Réal Ouellet, $\mathrm{La}$ Colonisation des Antilles, p. 5-27.

46 Philip Boucher, Cannibal Encounters, p. 42. Voir aussi Benoît Roux, «Le prêtre et le Callínago », p. 78-79. Auroux et Queixalos, « La première description linguistique », p. 109. Giovanni Pizzorusso, Roma nei Caraibi l'organizzazione delle missioni cattoliche nelle Antille e in Guyana (1635-1675), Rome, École française de Rome, 1995; id., «Propaganda fide e le missioni cattoliche sulla frontiera politica, etnica e religiosa delle Antille nel XVII secolo », Mélanges de l'École française de Rome Italie et Méditerranée, vol. 109, nº 2, 1997. Joseph Rennard, Histoire religieuse des Antilles françaises des origines à 1914 d'après des documents inédits, Paris, 1954.

47 Philip Boucher, Cannibal Encounters, p. 53.

48 Marie-Christine Gomez-Géraud affirme que le voyage de Jean de Léry au Brésil devient vite le bestseller français au sujet de l'Amérique, tandis que l'intérêt pour Thevet et ses 
Les relateurs des îles adressent essentiellement leurs textes aux futurs missionnaires et s'appuyaient, au-delà des textes émanant des membres de leurs propres ordres, sur un vaste corpus de témoignages portant sur la région antillaise et sud-américaine ; en particulier ceux concernant le Brésil, en raison des échanges entre ce pays et les Îles. Les travaux de Raymond Breton servent de référence à presque tous les voyageurs. Quand il publie finalement ses propres ouvrages chez Bouquet à Auxerre, le Dictionnaire caraïbe françois meslé de quantités de remarques historiques pour l'esclaircissement de la langue en 1665 et l'année suivante une Grammaire caraïbe, il affirme explicitement que ses textes sont destinés aux missionnaires. À en croire l'adresse aux révérends pères missionnaires qui préface le Dictionnaire Caraïbe-François de Breton, il serait lui-même l'auteur de presque toutes les descriptions des Amérindiens des Îles. « I'écrivis donc les 10 chapitres des Sauvages qu'on a suivis depuis», rappelle Breton avant de dresser la liste de ceux qui s'en sont particulièrement servis. Parmi eux, Du Tertre :

I'ay donné aux pressentes importunités du R. P. du Tertre (qui s'est dignement acquitté du devoir d'historien des Antiles) une parcelle de mes traductions de Sauvage en Latin, mais il ne les agréa pas, il voulut quelque chose en langue vulgaire qui fit connaîstre l'imperfection de la langue Caraïbe, ce qui m'obligea de changer la traduction Latine, en construction Française qu'il arrangea à la fin de son livre comme une traduction. Je lui donnai pour un essai de la langue et non pas pour une chose orthodoxe quoiqu'on dise du Français ou de la glose, le texte Caraïbe me semble bon, ceux qui auront passé le jargon des enfants et les dialectes des femmes, le connaîtront avec le temps, s'ils lui donnent sa vraie prononciation ${ }^{49}$.

Il n'est pas anodin que ce soit à Du Tertre que Breton confie ses manuscrits, sa relation et son dictionnaire. C'est grâce à l'arrivée aux Îles de Du Tertre en 1640 que Breton peut poursuivre son travail d'évangélisation des Autochtones, on l'a noté plus haut. Or, il n'est pas le seul à utiliser les sources de Breton.

singularités s'épuise et que les relations de Jacques Cartier passent presque inaperçues au XVI ${ }^{\mathrm{e}}$ siècle; Écrire le voyage au XVI ${ }^{e}$ siècle en France, Paris, Presses universitaires de France, 20oo, p. 9. Pour satisfaire un public plus séduit par l'Orient, on imprime en France « deux fois plus de livres sur les Turcs et l'Empire turc, que sur l'Amérique », p. 10. Henri-Jean Martin confirme que l'Orient demeure la source d'inspiration principale de l'imaginaire du lointain au XVII ${ }^{\mathrm{e}}$ siècle : Livre, pouvoirs et société à Paris au XVII ${ }^{e}$ siècle (1598-1701), Genève, Droz, 1969, p. 207.

49 Breton, Dictionnaire Carä̈be-Français, p. vi. L'orthographe et la ponctuation ont été modernisées dans cette édition. 
Avant de mentionner Du Tertre, Breton évoque en effet Mathias Du Puis qui aurait écrit, toujours d'après Breton, la première description de la région, publiée chez Martin Yvon à Caen en 1652, Relation de l'establissement d'une colonie francoise dans la Gardeloupe isle de l'Amerique, et des mours des sauvages ${ }^{50}$. En réalité, Du Puis n'est pas le premier. Le jésuite Jacques Bouton avait déjà publié une Relation de l'establissement des François depuis l'an 1635. En l'Isle de la Martinique, l'une des ant-isles de l'Amérique, chez Cramoisy, en 1640, l'année même où Bouton débarque à la Martinique ${ }^{51}$. Les principaux ouvrages, en dehors de celui de Du Puis, sont la Relation des missions des PP. de la compagnie de Jesus Dans les Isles, \& dans la terre ferme de l'Amérique Meridionale $(1655)^{52}$ de Pierre Pelleprat, missionnaire jésuite également auteur d'une Introduction à la langue des Galibis, sauvages de la terre ferme $(1655)^{53}$. Du Tertre compose la deuxième édition de son ouvrage en se référant à ces relations, notamment à celles qui critiquent directement ou indirectement sa position quant à la mission et à la politique coloniale. Voyage de la France equinoxiale en l'isle de la Cayenne d'Antoine Biet, curé de l'église Sainte-Geneviève parti pour Cayenne en 1651 , en est un bon exemple ${ }^{54}$. Dans le chapitre sur la vie des habitants et le fonctionnement des missions, Du Tertre revient sans cesse à cet ouvrage pour réfuter la critique des dominicains, à qui Biet reproche la conversion trop facile des Amérindiens. Du Tertre, de son côté, rétorque que le curé en juge trop hâtivement, faute d'une connaissance assez approfondie de la vie dans les Îles.

À partir de 1670, les ouvrages antillais se font encore moins nombreux ${ }^{55}$. Le Jardin du Roi envoie des voyageurs dans les Îles pour enrichir les connaissances

$5^{\circ}$ Mathias Du Puis, Relation de l'establissement d'une colonie francoise dans la Gardeloupe isle de l'Amerique, et des moeurs des sauvages, Caen, Martin Yvon, 1652. Voir aussi Breton, Dictionnaire Carä̈be-François, article 12, NP.

51 Jacques Bouton, Relation de l'establissement des François depuis l'an 1635 en l'isle de la Martinique, l'une des isles de l'Amerique, des mours des sauvages, de la situation et des autres singularitez de l'isle, Paris, Cramoisy, 1640.

52 Pierre Pelleprat, Relation des missions des PP. de la compagnie de Jesus dans les Isles, \& dans la terre ferme de l'Amérique Meridionale, Paris, S. et G. Cramoisy, 1655. Mentionnons ici une publication moins connue, peut-être parce que l'auteur parle des îles Camercanes et non pas des Ant-Isles, Voyage des îles Camercanes en l'Amérique quifont partie des Indes occidentales, du carmélite Maurile de Saint-Michel, publié au Mans chez Hierôme Olivier en 1652.

53 Pierre Pelleprat, Introduction à la langue des Galibis, sauvages de la terre ferme, Paris, S. \& G. Cramoisy, 1655 .

54 Antoine Biet, Voyage de la France équinoxiale en l'isle de la Cayenne, entrepris par les François en MDCLII, Paris, Clouzier, 1664.

55 Une publication importante - Relation de l'origine, mours, coutumes, religions, guerres et voyages des Caraïbes sauvages des Isles Antilles de l'Amérique, écrite par Le Sieur de La Borde - apparaît illustrée dans un volume édité par Henri Justel, Receuil de divers 
en botanique, mais il n'en sort que deux ouvrages de Charles Plumier vers la fin du XVII ${ }^{\mathrm{e}}$ et au début du XVIII ${ }^{\mathrm{e}}$ siècles $^{56}$. À la même époque, pourtant, les récits de flibuste connaissent un succès remarquable, à l'image de l'Histoire des aventuriers flibustiers de l'Amérique d'Alexandre Exquemelin, publiée d'abord en néerlandais en 1668, puis en français en 1686. La traduction française de ce livre emprunte des passages entiers, par exemple la description de lî̂le de la Tortue, à Du Tertre. Le texte d'Exquemelin sera réédité et publié avec d'autres récits de flibustiers dans le Journal du voyage fait à la mer du Sud avec les flibustiers de l'Amérique en 1684 et les années suivantes de Jacques Raveneau de Lussan, paru en $169 \mathrm{o}^{57}$. C'est dans les dernières décennies du Grand Siècle marquées par la vogue de la flibuste que le dernier relateur de cette période coloniale antérieure à la montée de l'économie esclavagiste dans les plantations part aux Antilles : le dominicain Jean-Baptiste Labat, qui résida en Martinique entre 1694 et 1706. Son Nouveau voyage aux Isles de l'Amérique: contenant l'histoire naturelle de ces pays, l'origine, les mours, la religion et le gouvernement des habitants anciens et modernes n'est cependant publié qu'en 1722. Beaucoup de passages relatifs à l'histoire de l'établissement aussi bien qu'à l'histoire naturelle sont pour une grande partie construits sur l'histoire de Du Tertre, que Labat reprend et corrige parfois ${ }^{58}$.

Le seul ouvrage écrit par un voyageur de l'établissement qui puisse rivaliser avec le livre de Du Tertre par son ampleur et sa qualité littéraire est l'Histoire naturelle et morale des Isles Antilles de l'Amérique du protestant Charles de Rochefort, publiée à Rotterdam en $1658^{59}$. Ce sont les seuls livres illustrés sur les Antilles du début de la colonisation, et les éditeurs de la Bibliothèque historique de la France les placent en tête de la liste des ouvrages sur les Îles, comme les publications les plus complètes sur le sujet ${ }^{60}$. Les premières éditions $\left(165^{8}\right.$, 1665, 1681 et 1716) de l'Histoire morale et naturelle des Isles Antilles sont publiées

voyages faits en Afrique et en l'Amérique qui n'ont point esté publiez, Paris, Chez Louïs Billaine, 1674 .

56 Charles Plumier, Description des plantes de l'Amérique; Id., Traité des fougères de l'Amérique, Paris, Imprimerie royale, 1705 .

57 Alexandre Exquemelin, Histoire des aventuriers flibustiers de l'Amérique, Paris, La Découvrance, 2012 [1686] ; Raveneau de Lussan, Journal du voyage fait à la mer du Sud avec les flibustiers de l'Amérique en 1684 et les années suivantes, Paris, Jean-Baptiste Coignard, 1690.

58 Jean-Baptiste Labat, Nouveau voyage aux îles de l'Amérique.

59 Charles de Rochefort, Histoire naturelle et morale des Isles Antilles de l'Amérique, Rotterdam, Arnould Leers, 1658.

6o Bibliothèque historique de la France, contenant le catalogue des ouvrages, imprimés \& manuscrits, qui traitent de l'histoire de ce royaume, ou qui y ont rapport; avec des notes critiques et historiques, vol. 3, Jacques Le Long et al. (dir.), Paris, Herissant 1771, p. 664-665. 
sans le nom de leur auteur. L'ouvrage a été attribué tantôt à Du Tertre, tantôt à Breton, tantôt au lieutenant général des Antilles de Poincy, tantôt à César de Rochefort. On voit en 1666 paraître à Paris, chez Louis Billaine - d'ailleurs un concurrent de Thomas Jolly, l'éditeur de Du Tertre - une Relation de l'Isle de Tabago, ou de la nouvelle Oüalcre, l'une des Isles Antilles de l'Amerique écrite par le Sieur de Rochefort ${ }^{61}$. Du Tertre, quant à lui, n'hésite pas à écrire dans l'avis au lecteur de l'édition de 1667 que de Rochefort est bien l'auteur de l'Histoire morale et naturelle des Isles Antilles.

L'affaire n'est pas simple pourtant, car l'ouvrage de de Rochefort fait l'objet d'une accusation de plagiat. Dès 1654, Du Tertre l'incrimine en effet, dans son épître dédicatoire à Harlay, de lui avoir volé son manuscrit, affirmant que la préparation de l'Histoire morale et naturelle des Isles Antilles l'aurait obligé à publier la première version de son ouvrage alors que son aventure aux Antilles nétait pas encore terminée. Dans son adresse à Harlay, Du Tertre souligne que la « présente » édition (celle de 1654, donc) a été achevée à la hâte afin qu'elle puisse voir le jour avant l'ouvrage de son rival :

[Je] n'aurois iamais pensé à le donner au public, si ie n'avois été averty qu'il étoit plus mal-heureux sur la terre que sur la mer, \& qu'apres avoir évité les Pirates de dix-huit cens lieüs de mer, il étoit tombé entre les mains de certains autres Pirates, qui font profession de s'enrichir des pertes d'autruy, \& qu'ils vouloient mettre au iour sous leur nom, encore qu'ils n'en eussent qu'une copie forte imparfaite. (1654: NP)

Ce piratage hypothétique inscrit l'ouvrage dans un imaginaire caribéen bien propre à « vendre » comme un livre d'aventures l'histoire que l'on va lire. Mais le passage fait aussi référence indirectement au parcours du ministre protestant, qui a en effet servi à l'île de la Tortue, le repaire des flibustiers américains. De Rochefort, de son côté, affirme dans son avis au lecteur s'être rendu à Paris autour de 1650, en quête d'un protecteur pour son projet d'ouvrage ; en vain. Il a pu lire le manuscrit de Du Tertre, qui circulait dans la capitale à cette époque. Cela expliquerait l'allusion faite par ce dernier relativement au fait que l'Histoire de la Guadeloupe aurait été «plus mal-heureux sur la terre que sur la mer ».

Les illustrations sont plus nombreuses dans l'ouvrage de de Rochefort, mais elles sont moins élaborées que celles de l'édition de 1667 de Du Tertre.

61 Sieur de Rochefort, Relation de l'Isle de Tabago, ou de la nouvelle Oüalcre, l'une des Isles Antilles de l'Amerique, Paris, Louis Billaine, 1666. 
Du Tertre revient à l'affaire du plagiat dans l'édition de 1667 , mais cette fois en en précisant les circonstances et en nommant Charles de Rochefort. Entre-temps, le protestant a déjà publié la deuxième édition de l'Histoire naturelle et morale des Isles de l'Amérique, dédiée au gouverneur de lîle de Tobago, Monsieur de Beveren, et une troisième édition paraîtra en 1667, celle-ci dédiée à Camille de Nevfville, archevêque et comte de Lyon. Cette fois, Du Tertre explique que le lieutenant général de Poincy aurait prié Raymond Breton de donner ses notes sur la langue des Amérindiens à une personne inconnue, qu'il a pu identifier plus tard comme étant de Rochefort ${ }^{62}$. De Poincy est cependant mort depuis 166o, et son administration libre des Îles ne pouvait guère lui avoir attiré de soutien, ni du côté du roi ni de celui de Colbert. Du Tertre peut donc sans crainte accuser de Poincy, connu par ailleurs comme un ami des protestants aux Îles. Breton confirme effectivement que c'est à lui qu'il avait confié ses notes :

Monsieur Aubert qui succéda au gouvernement de l'île à Mr. de l'Olive m'écrivit de Flexingue, et me pria de la part de Mr. de Poincy Lieutenant général pour le Roi sur les Isles, de donner au porteur de la sienne des mémoires, si c'est l'auteur même de la relation Imprimée à Rotterdam en 1658 je n'en sais rien, tant y a que je lui en donnai qu'il n'a suivi qu'autant qu'il a voulu, et parce qu'il ne me les attribue pas en particulier comme le vocabulaire je les laisse passer et réponds que je lui fis écrire et ponctuer en ma présence le vocabulaire, et je le confesse mien à la réserve des mots de banaré, manigat, carebet, aioupa, Amac, coüi, mouchache, cacone, coincoin, maron, piknine, boucan, Tortille, pisquet \& canari qui ne sont point mots Sauvages, et qui ne viennent point de moi; ceux qui les lui ont donné les peuvent bien avoir ouï-dire aux Sauvages, et aux Français mais comme un jargon pour se faire entendre, et non pour un véritable langage Caraïbe ${ }^{63}$.

Breton se plaint de ne pas être reconnu comme la source de l'ouvrage de de Rochefort. Il le corrige donc, mais sans prendre clairement position sur la question du vol. Du Tertre est plus précis, disant que de Rochefort avait reçu les notes de Breton et la première édition de l'Histoire générale des Antilles en

62 Pour l'affaire des manuscrits de Breton, voir l'article d'Odile Gannier « Le tupi et le galibi sans peine : Glossaires, manuels et catéchismes à l'usage des voyageurs et missionnaires (XVI ${ }^{\mathrm{e}}-\mathrm{XVII}{ }^{\mathrm{e}}$ siècles) », Échos des textes, échos des voix : Étude sur le dialogue, en hommage à Béatrice Périgot, Odile Gannier et Véronique Montagne (dir.), Paris, Classiques Garnier, 2013, p. 14 ; et Auroux et Queixalos, « La première description linguistique », p. 111.

63 Breton, Dictionnaire Caraïbe-François, p. vii. 
même temps, ce qui aurait retardé la publication de l'Histoire naturelle et morale des Isles de l'Amérique, qui ne sortira donc qu'en 1658. Or, en 1667, quand il a finalement lu le livre de son rival, le vol semble moins l'incommoder que les imperfections du livre de ce dernier:

Ce Livre [celui de de Rochefort] fut incontinent presenté à Messieurs de l'Assemblée des Phisiciens, Mathematiciens, \& Astronomes, qui apres en avoir loüé le discours, remarquerent qu'excepté les digressions qu'il a faites, tres-peu convenable à l'histoire des Ant-Isles, le vocabulaire de ce bon Pere, \& les belles Antitheses de ses Amis, presque tout le reste estoit si fidellement tiré de mon Livre, qu'il n'a pas mesme obmis les fautes que j'y avois faites. (1667: NP)

Du Tertre affirme ainsi le plagiat sous le couvert des autorités savantes qui se rassemblaient alors chez Montmor: seules les parties de l'ouvrage de de Rochefort qui traitent des Amérindiens et des animaux en Amérique du Nord ne correspondraient pas à son manuscrit de 1648 . Qui plus est, le propos indique que les montmoriens auraient changé d'avis sur le travail du protestant. Découvrant les emprunts que celui-ci aurait faits à Du Tertre, Monsieur de Montmor lui-même l'aurait prié de procéder à une réédition de son propre livre, qu'il ferait imprimer en Hollande à ses propres frais. Notre missionnaire fit savoir qu'il avait été prêt à le faire, mais qu'il avait finalement abandonné ce projet, préférant prendre plus de temps et inclure dans une nouvelle édition d'autres observations faites à partir de son retour aux Antilles en 1656, où il avait eu l'occasion de visiter toutes les îles.

De Rochefort avait sans doute lu Du Tertre. Certains passages se présentent effectivement comme des copies de l'ouvrage du dominicain, mais il ne s'agit pas d'une reprise intégrale. Dans un premier temps, de Rochefort insère de longues digressions sur d'autres peuples et régions, comme les Appalaches et les Amérindiens de la Nouvelle-France. Or, chercher si loin les origines des habitants naturels des Îles n'est, selon Du Tertre, qu'un prétexte pour « grossir son livre d'histoire, que $\mathrm{M}^{\mathrm{r}}$ Bristol a fait des Apalachites » (1667, tome II : 363 ), l'accusant ainsi d'un double plagiat. Des différences d'interprétation se font jour sur plusieurs événements : c'est notamment le cas dans le récit que les deux auteurs font de la guerre entre les Français et les Amérindiens en 1632. Selon de Rochefort, les Amérindiens sont responsables du conflit ${ }^{64}$; Du Tertre, de son côté, blâme les Français et Monsieur de l'Olive. Leurs observations de la

64 Charles de Rochefort, Histoire naturelle et morale des Isles Antilles, p. 282. 
nature sont également traitées différemment du point de vue stylistique; surtout, elles se fondent sur des conceptions de savoir divergentes. Le protestant a bel et bien séjourné au moins deux fois aux Îles (ce dont Du Tertre convient d'ailleurs), et c'est à tort qu'on lui reproche d'être un voyageur de cabinet. Mais à la différence de Du Tertre, il y a peu de traces de sa propre expérience dans l'Histoire naturelle et morale des Isles Antilles; sa conception du savoir reste livresque et traditionnelle. On peut aussi constater que Du Tertre fait souvent allusion à l'ouvrage de de Rochefort dans son texte, alors que le protestant ne mentionne qu'indirectement l'ouvrage de ce dernier, essentiellement dans la préface et dans l'avis au lecteur dans les diverses éditions qui suivent celle de 1658 .

Étant donné que ce sont surtout les catholiques qui écrivent sur les Antilles françaises, il n'est guère surprenant de constater que Du Tertre est une référence plus commune dans le corpus des voyages antillais que de Rochefort. De plus, de Rochefort ne s'inscrit pas dans le projet colonial au même degré que Du Tertre, et il est moins engagé dans la vie quotidienne des habitants des Îles. $\mathrm{Au} \mathrm{XVIII}^{\mathrm{e}}$ siècle, quand les sources antillaises sont consultées dans le cadre des sciences naturelles, notamment la botanique et la géographie, c'est surtout à Du Tertre que l'on se fie. Dans l'Encyclopédie de Diderot et d'Alembert, par exemple, Du Tertre est cité 26 fois, tandis que de Rochefort n'est mentionné que 4 fois. Encore aujourd'hui, les catalogues des bibliothèques attribuent aujourd'hui l'Histoire naturelle et morale des Isles de l'Amérique à Du Tertre autant qu'à de Rochefort ou encore à Raymond Breton. Selon la notice du catalogue de la Bibliothèque Mazarine, c'est précisément le manuscrit incomplet à la Bibliothèque nationale de France qui aurait été volé et ensuite imprimé sous le nom de de Rochefort.

La version que donne Du Tertre de l'affaire en 1667 a donc convaincu, malgré le fait que le livre de de Rochefort - traduit en néerlandais, en anglais et en allemand, et réédité quatre fois entre 1665 et 1716 - a été plus répandu que le sien à l'époque de sa publication ${ }^{65}$. On confond toujours les deux en faveur de Du Tertre. Michèle Duchet suggère par exemple dans son Anthropologie au siècle des Lumières que la comparaison entre les Caraïbes et les Amérindiens de l'Amérique du Nord serait à porter au crédit de Du Tertre, bien qu'elle appartienne en réalité à de Rochefort ${ }^{66}$. Certains lecteurs, comme Cornelius de

65 Pour un tableau complet des éditions et des traductions du livre de de Rochefort, voir Benoît Roux, «Le Pasteur Charles de Rochefort et l'Histoire naturelle et morale des îles Antilles de l'Amérique », p. 176 et 193. 
Pauw, critiquent la fiabilité de l'histoire de de Rochefort; d'autres, comme Claude Lévi-Strauss dans son discours inaugural au Collège de France, oublient tout simplement de le mentionner à côté des grands historiens des Antilles du $\mathrm{XVII}^{\mathrm{e}}$ siècle $^{67}$. Cependant, le but de cette étude n'est ni de résoudre l'énigme de l'affaire du plagiat ni de comparer ces deux ouvrages. Ce qui importe pour la suite de l'analyse, c'est le constat que la manière dont Du Tertre se rapporte à de Rochefort a des effets certains sur les figurations de l'étranger.

67 Benoît Roux, «Le Pasteur Charles de Rochefort et l'Histoire naturelle et morale des îles Antilles de l'Amérique», p. 176. 